\title{
Les gîtes à limnées dans les prairies marécageuses sur sol acide : les effets d'impacts agricoles
}

\author{
L. Vareille ${ }^{1}$ \\ C. Vareille-Morel2 \\ G. Dreyfuss ${ }^{1}$ \\ D. Rondelaud ${ }^{3}$
}

\begin{abstract}
Mots clés : Mollusques, Lymnaea sp., Physa acuta., Potamopyrgus antipodarum, prairie, Limousin.
\end{abstract}
Des investigations malacologiques ont été réalisées en 1974 et en 1994-1995 dans trois prairies de la Haute-Vienne afin de préciser les caractéristiques des gîtes à limnées lorsque les parcelles sont abandonnées ou modifiées par l'application de mesures agronomiques. L'abandon d'une prairie et l'invasion de ragondins se sont traduits par des modifications dans la distribution des limnées. Lymnaea cf. fuscus a déserté le fossé principal de drainage pour coloniser les rigoles et les couloirs créés par ces rongeurs, ce qui s'est traduit par un accroissement important de la superficie du nouveau gîte. Les effectifs moyens de $L$. cf. fuscus et de L. truncatula ont augmenté aux dépens de ceux de L. glabra. Un autre mollusque aquatique, Physa acuta, a envahi le fossé laissé libre. Le curage des rigoles dans les deux autres prairies et leur invasion par une ou deux espèces aquatiques ( $L$. ovata, Potamopyrgus antipodarum ou Physa acuta) se sont traduits par une extension dans la superficie du gîte aussi bien pour les mollusques colonisateurs que pour $L$. glabra et $L$. truncatula. Les effectifs des trois premières espèces sont assez importants dans ces deux rigoles ; par contre, on note une réduction du nombre des deux limnées.

\section{Lymnaeid habitats in swampy meadows on acid soil : effects of agricultural impacts}

Characteristics of lymnaeid habitats were studied in three swampy meadows on acid soil i) in 1974, and ii) in 1994 or 1995 after disturbance related to cessation or modification of agricultural practices. Changes in the distribution of three Lymnaea species were noted in the first meadow after its abandon with an invasion of Myocastor coypus. Desertion of the main drainage furrow and colonization of ditches and rodent passageways by $L$. cf. fuscus were detected in 1994, inducing an increase in the overall area of snail habitat. The mean numbers of $L$. cf. fuscus and $L$. truncatula were greater, whereas those of $L$. glabra slightly decreased. Another aquatic snail, Physa acuta, had invaded the unoccupied drainage furrow. Clearing of drainage ditches in the other two meadows, followed by the invasion in 1995 of one or two aquatic species (Lymnaea ovata, Potamopyrgus antipodarum, Physa acuta), induced an increase in the habitat area for invading snails as for $L$. glabra and $L$. truncatula. The numbers of the first three species were high in the two ditches; in contrast, there was a reduction in the numbers of the two lymnaeid species.

Key words : Mollusca, Lymnaea sp., Physa acuta., Potamopyrgus antipodarum, meadow, Limousin.

\section{Introduction}

Quatre espèces de Lymnaea peuvent se rencontrer dans les prairies marécageuses sur sol acide et occupent des zones précises sur le réseau de drainage superficiel. Lymnaea truncatula (Müller) colonise l'extrémité distale des rigoles et $L$. glabra (Müller) se rencontre sur le cours moyen de celles-ci. Les gîtes de $L$. palustris (Müller), lorsque cette espèce est présente; se

1. Faculté de Pharmacie, Laboratoire de Parasitologie, 2, rue du Docteur-Raymond-Marcland, 87025 Limoges Cedex.

2. Faculté des Sciences, Laboratoire de Malacologie Appliquée, 123, avenue Albert-Thomas, 87060 Limoges Cedex.

3. Faculté de Médecine, Laboratoire d'Histopathologie Parasitaire, 2, rue du Docteur-Raymond-Marcland, 87025 Limoges Cedex.

Article available at http://www.limnology-journal.org or http://dx.doi.org/10.1051/limn/1996012 situent dans la partie inférieure du fossé principal tandis que $L$. ovata (Draparnaud) vit dans le ruisseau situé plus en aval (Bouix-Busson \& Rondelaud 1984, Mage \& Rondelaud 1991). D'après Økland (1990), cette distribution des colonies dans une prairie dépend de la compétition entre les espèces.

Une note antérieure précise les caractéristiques de ces habitats et les effectifs du mollusque dans 53 prairies marécageuses de la Haute-Vienne (Vareille 1996). Si l'on considère les gîtes de $L$. truncatula $(4,3$ en moyenne par prairie), on constate que leur superficie ne dépasse pas $5 \mathrm{~m}^{2}$ par gîte et que les effectifs du mollusque au mois de juin se situent entre 32 et 83 individus selon le secteur géographique étudié. Les habitats de $L$. glabra sont moins nombreux $(2,4$ par prairie), de superficie plus importante (jusqu'à $7,2 \mathrm{~m}^{2}$ ) et l'effectif 
des colonies plus important ( 81 à 187 individus selon le secteur).

Nous nous sommes posé la question de savoir si les caractéristiques de ces gîtes persistent lorsque les prairies marécageuses subissent une transformation majeure dans leur topographie. Les trois exemples de pâtures que nous rapportons dans cette note portent sur l'une des modifications suivantes : abandon de la prairie et invasion de celle-ci par des ragondins, curage récent du système de drainage, captage d'une source et déversement dans une rigole de drainage. Les décomptes de mollusques ont été réalisés dans ces parcelles en 1974 (avant les transformations) et en 1994 ou 1995.

\section{Matériel et méthodes}

\subsection{Stations d'étude}

- La première prairie se situe au lieu-dit Le Châtenet, commune de Feytiat (Haute-Vienne). Elle longe un ruisseau et présente un système de drainage superficiel avec un fossé principal long de $300 \mathrm{~m}$ environ et sept rigoles plus longues au sud qu'au nord $(80 \mathrm{~m}$ au lieu de 10 à $15 \mathrm{~m}$ ). Un second fossé de drainage a été creusé en 1988. Trois espèces de limnées : $L$. cf. fuscus (Pfeiffer), L. glabra et $L$. truncatula, colonisaient le réseau en 1974. L'abandon de cette prairie à partir de 1986 s'est traduit par la disparition de la jonchaie et par le développement d'une caricaie à Carex rostrata (Stockes) dans la partie la plus déclive. Des ragondins, Myocastor coypus (Molina), ont envahi le ruisseau à partir de 1992 et ont créé des couloirs dans la végétation de cette prairie pour aller se nourrir. Le fossé de drainage a alors été envahi par Physa acuta (Draparnaud) à partir du ruisseau tandis que la colonie de $L$.cf. fuscus s'est déplacée dans les rigoles ou les couloirs créés par les ragondins.

- La deuxième parcelle est localisée au lieu-dit Le Marchadeau, commune de Burgnac (Haute-Vienne). Elle est traversée par un ruisseau dans lequel se jette une rigole longue de $100 \mathrm{~m}$ environ. En 1974, le ruisseau était colonisé par le prosobranche Potamopyrgus antipodarum (Gray) tandis que les rigoles abritaient $L$. glabra et $L$. truncatula. Le curage du fossé en 1993, le rejet de la vase sur les terres émergées et.la rénovation partielle des deux rigoles se sont traduits par un déplacement de $P$. antipodarum vers la rigole.

- La troisième station se situe au lieu-dit La Roseraie, commune de Limoges et longe la rivière de l'Aurence. Elle comprend un fossé principal de $100 \mathrm{~m}$ de long et deux rigoles de drainage superficiel longues de 80 et $10 \mathrm{~m}$. Les rigoles étaient colonisées par L. glabra et $L$. truncatula en 1974. La rénovation du système de drainage, le captage d'une source permanente et son déversement dans l'une des rigoles en 1985 se sont traduits par l'envahissement du réseau par deux espèces (L. ovata, Physa acuta) à partir de la rivière.

Ces trois prairies subissent un dessèchement estival de 15 jours à 1 mois. Mais il persiste toujours un peu d'eau stagnante dans le fossé de la première prairie, dans la partie la plus basse des deux rigoles pour la seconde et dans le fossé pour la dernière parcelle.

\subsection{Protocole des observations}

Les investigations dans ces trois prairies ont été réalisées a) en juin 1974 (avant les modifications agronomiques), b) en juin 1994 ou juin 1995. Les deux premiers paramètres sont le nombre de gîtes pour chaque espèce et la superficie de chaque habitat. Le troisième facteur concerne l'effectif de chaque colonie.

Le dénombrement des mollusques a été réalisé sur le système de drainage de chaque prairie en utilisant des quadrats de deux mètres carrés dans la prairie de Feytiat et d'un mètre carré dans les deux autres pâtures. Le nombre de zones échantillonnées est de 30 dans la prairie de Feytiat et ces quadrats ont été disposés les uns à la suite des autres pour couvrir la partie centrale du fossé et la longueur totale de trois rigoles. Il est de 10 et 9 respectivement dans les deux autres stations et les quadrats sont disposés à intervalles réguliers (6 ou 8 mètres) sur la longueur totale de la rigole. Un décompte a également été effectué en 1994 et 1995 sur la longueur totale de 15 couloirs créés par les ragondins dans la prairie de Feytiat.

Les animaux ont été récoltés par chasse à vue sur chaque quadrat sur les zones exondées, aussi bien sur le sédiment que dans la végétation présente. Les prospections dans chaque quadrat ont été réalisées successivement par deux personnes, avec une durée de 20 à 30 minutes par zone échantillonnée. Les espèces ont été ensuite déterminées directement sur le terrain, à l'exception des juvéniles (hauteur $<3 \mathrm{~mm}$ ) qui sont rapportés au laboratoire et identifiés sous loupe binoculaire.

Les valeurs individuelles recueillies dans la prairie de Feytiat ont été exprimées sous forme de moyennes et d'écarts types pour une rigole, en tenant compte de l'année d'étude, du numéro d'ordre du quadrat, de l'espèce de limnée et de la nature du paramètre.

La superficie totale des gîtes a été déterminée dans chaque prairie pour chaque espèce en 1974 et en 1995. L'effectif de chaque espèce a été établi en multipliant la superficie du gîte correspondant par l'effectif moyen relevé dans les quadrats échantillonnés. Les résultats obtenus en 1995 figurent sur le tableau I. 


\section{Résultats}

\subsection{Prairie de Feytiat}

La figure $1 \mathrm{~A}$ indique les effectifs des limnées en 1974. Les $L$. cf. fuscus sont présentes au niveau du fossé central (quadrat 1) et à l'embouchure des rigoles (quadrat 2). L'effectif moyen de la colonie est donc de 65 individus sur $4 \mathrm{~m}^{2}$. Les $L$. glabra ont été observées sur le cours moyen des rigoles et leur effectif est en moyenne de 63,8 individus sur $8 \mathrm{~m}^{2}$ (quadrats $4,5,6$ et 7). Les $L$. truncatula sont présentes à l'extrémité terminale des mêmes rigoles (quadrats 9 et 10) et les effectifs des colonies sont assez faibles. Les trois espèces de limnées occupaient des emplacements précis sur le système de drainage superficiel en juin 1974. La figure $2 \mathrm{~A}$ visualise les aires occupées par ces diverses colonies. La colonie de $L$. cf. fuscus est unique ; par contre, on dénombre en général une colonie de $L$. glabra et une population de $L$. truncatula par rigole de drainage. Les colonies des deux dernières espèces dépendent donc du nombre de rigoles de drainage présentes dans la prairie.

La figure 1B montre les effectifs des limnées sur les mêmes zones en 1994. L. cf. fuscus a pratiquement déserté le fossé central (3,5 individus dans le quadrat 1$)$; par contre, le mollusque a colonisé les rigoles sur toute leur longueur avec une densité maximale dans les quadrats 2 et 3 . L'effectif de la colonie est de 116 individus par rigole (sur $20 \mathrm{~m}^{2}$ ). La population de $L$. glabra s'est étendue sur toute la rigole mais leur effectif est plus faible : 47,5 individus en moyenne sur $18 \mathrm{~m}^{2}$ (quadrats 2 à 10 ). L' habitat colonisé par $L$ truncatula est plus vaste ( $18 \mathrm{~m}^{2}$ environ par rigole); l'effectif est en légère augmentation (50 individus en moyenne) mais la densité maximale se situe toujours à l'extrémité aveugle des rigoles (quadrats 9 et 10 ).

Ces modifications dans la localisation des limnées et leur nombre se retrouvent également dans les $15 \mathrm{cou}-$ loirs creusés dans la végétation. Les $L$. cf. fuscus et $L$. truncatula ont des effectifs plus faibles que ceux rapportés dans les rigoles (35,4 et 26,2 ind respectivement sur $22 \mathrm{~m}$ de couloir). Le nombre des $L$. glabra y est très faible : moins de 5 individus par conduit (résultats non représentés).

Le fossé central a été envahi par une population de Physa acuta en provenance du ruisseau, avec un effectif de 24 à 46 individus (sur $2 \mathrm{~m}^{2}$ ) selon les quadrats. Des individus erratiques ont été observés à plusieurs reprises dans les rigoles mais ils ne résistent pas au dessèchement estival (résultats non représentés).

La figure $2 \mathrm{~B}$ précise la nouvelle localisation des limnées en juin 1994. La zone prairiale située à droite du
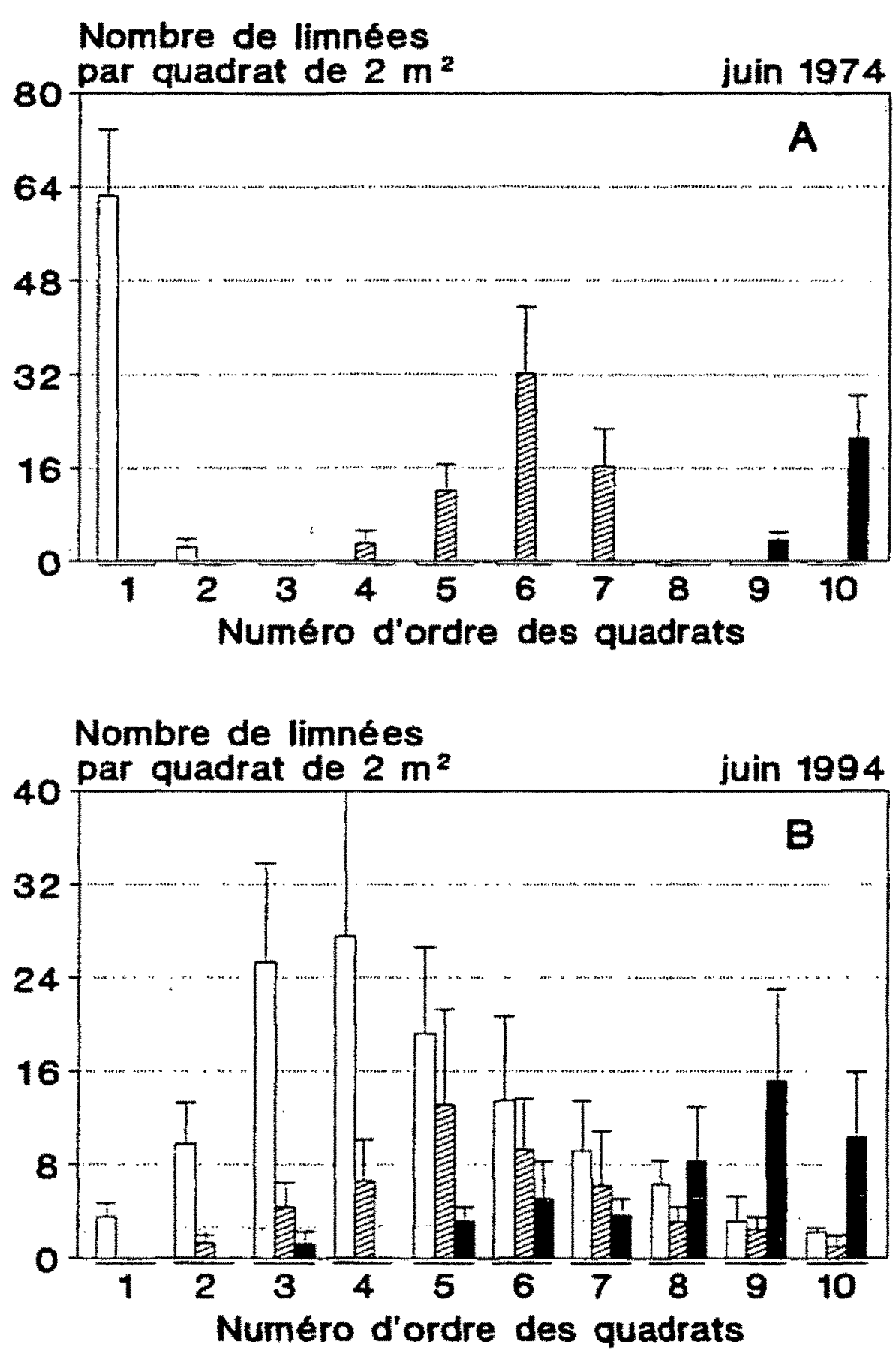

Lymnaea cf. fuscus
$L$. glabra

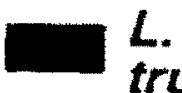

truncatula

Fig. 1. Station du Châtenet, commune de Feytiat (Haute-Vienne), Répartition numérique des limnées dans le fossé (quadrat $n^{\circ} 1$ ) et dans trois rigoles qui s'y jettent (quadrats $n^{\circ} 2$ à $n^{\circ} 10$ ) : en juin 1974 (A) et en juin 1994 (B). Les chiffres ont été obtenus sur des quadrats de $2 \mathrm{~m}^{2}$, depuis le fossé central jusqu'à l'extrémité aveugle des rigoles.

Fig. 1. Meadow of Châtenet, commune of Feytiat, department of Haute-Vienne, France. Numerical distribution of Lymnaea snails in the drainage ditch (quadrant $n^{\circ}$ ) and in three furrows (quadraints $n^{\circ} 2$ to $n^{\circ} 10$ ) : in June 1974 (A) and June 1994 (B). Snails were counted in $2-\mathrm{m}^{2}$ quadrants, from the central ditch to the peripheral zones of drainage furrows.

fossé est largement colonisée par les limnées tandis que les rigoles situées à gauche sont abandonnées. 

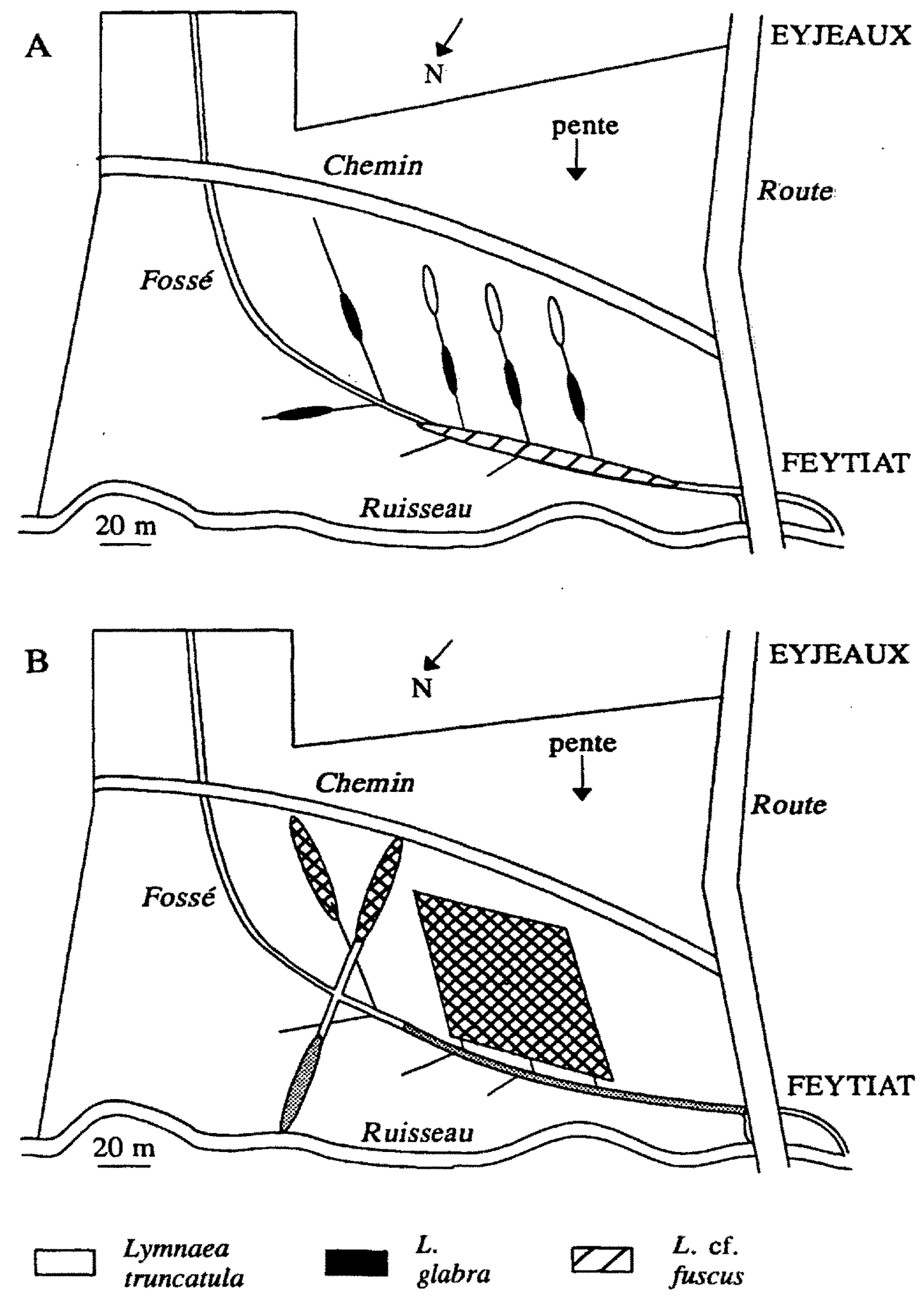

\section{Les 3 espèces , Physa}

Fig. 2. Localisation des différentes colonies dans la prairie de Feytiat en juin 1974 (A) et en juin 1994 (B). Fig. 2. Location of different snail populations in the Feytiat meadow in June 1974 (A) and June 1994 (B). 


\subsection{Prairie de Burgnac}

La figure 3 A précise les effectifs de $L$. glabra et de L. truncatula en juin 1974. L. glabra se situe dans le secteur aval de la rigole (quadrats 1 à 5). Le nombre de mollusques recensés sur ces zones ne dépasse pas 60 par quadrat. L. truncatula colonise le secteur amont. Les effectifs sont nettement plus faibles : moins de 20 limnées par quadrat.
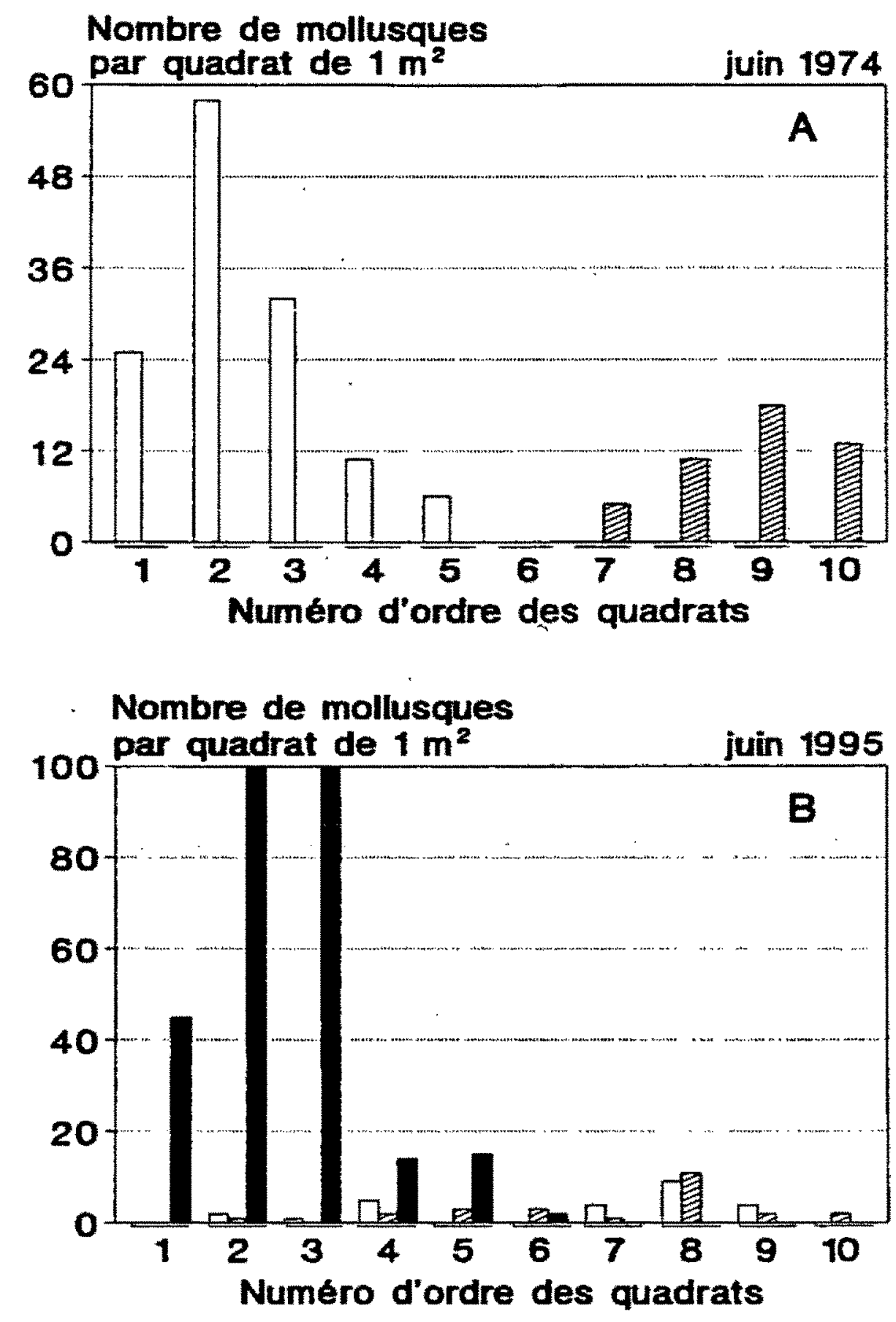

\section{Lymnaea} glabra
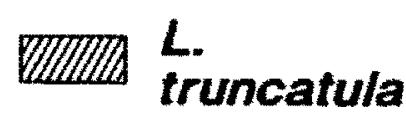

Potamopyrgus antipodarum

Fig. 3. Station du Marchadeau, commune de Burgnac (Haute-Vienne). Répartition numérique des limnées dans la rigole en juin 1974 (A) et juin 1995 (B). Les chiffres ont été obtenus sur dix quadrats de $1 \mathrm{~m} 2$, choisis à intervalles réguliers sur toute la longueur de la rigole.

Fig. 3. Meadow of Marchadeau, commune of Burgnac, department of Haute-Vienne, France. Numerical distribution of Lymnaea species in the drainage furrow in June 1994 (A) and June 1995 (B). Snails were counted in ten $1-\mathrm{m}^{2}$ quadrants, selected at regular intervals over furrow length.
Le curage du fossé en 1993 s'est traduit par une migration de Potamopyrgius antipodarum dans la rigole. L'extension de cette espèce a entraîné des modifications dans la localisation et les effectifs des deux limnées. Ces derniers ont été rélevés en juin 1995 (Fig. 3B). Le prosobranche a été retrouvé sur les six quadrats les plus proches du ruisseau. Ses effectifs sont assez élevés dans les trois premiers (de 45 à 101 mollusques) et plus faibles dans les trois suivants. Les deux limnées sont pratiquement présentes dans les neuf derniers quadrats et il n'y a plus de séparation nette entre leurs gîtes. Leurs effectifs sont faibles, ne dépassant pas $9 \mathrm{~L}$. glabra et $11 \mathrm{~L}$. truncatula dans le huitième quadrat.

\subsection{Prairie de Limoges}

La figure 4A précise les effectifs de deux limnées et leur distribution en juin 1974. L. glabra manque dans le secteur aval de la rigole (quadrats 1 à 3 ) mais s'observe sur le cours moyen de celle-ci (quadrats 4 à 7 ). Les effectifs par zone échantillonnée sont alors assez importants ( 27 à 44 limnées). La Limnée tronquée est présente dans les deux derniers quadrats avec de faibles nombres (moins de 15 mollusques par zone).

Des modifications portant sur le captage d'une source et son déversement dans la rigole ont été réalisées lors de la transformation de cette prairie en zone de loisirs. Lors de nos prospections en 1995, deux autres espèces de Pulmonés aquatiques ont été retrouvées (Fig. 4B). L ovata et Physa acuta (résultats non représentés) se rencontrent sur l'ensemble de la rigole mais elles présentent des variations d'effectif selon le quadrat. La densité maximale ne dépasse pas 20 individus par espèce dans chaque quadrat. Les deux autres limnées ont une distribution plus étendue qu'en 1974. $L$. glabra s'observe dans les deux premiers quadrats (secteur aval de la rigole) et sur les deux derniers. L. truncatula est présent dans les cinq derniers quadrats. Les effectifs de ces deux espèces dans chaque zone sont assez faibles.

3.4. Les caractéristiques des gîtes sur les prairies modifiées (Tableau 1)

La superficie des gites dans la prairie de Feytiat est largement augmentée pour les trois limnées indigènes: $179 \mathrm{~m}^{2}$ pour $L$. cf. fuscus par exemple au lieu de $23 \mathrm{~m}^{2}$ en $1974,89 \mathrm{~m}^{2}$ au lieu de $36 \mathrm{~m}^{2}$ pour L. truncatula. La physe en provenance du ruisseau a colonisé le fossé central sur la plus grande partie de sa longueur, soit $62 \mathrm{~m}^{2}$. Deux espèces ont présenté une explosion de leurs effectifs : $L$. cf. fuscus avec 873 individus sur l'ensemble du gîte en 1995 au lieu de 180 environ en 1974, L. truncatula avec 476 individus en 1995 au lieu de 176 en 1974. L'autre limnée, L. glabra, a subi une 

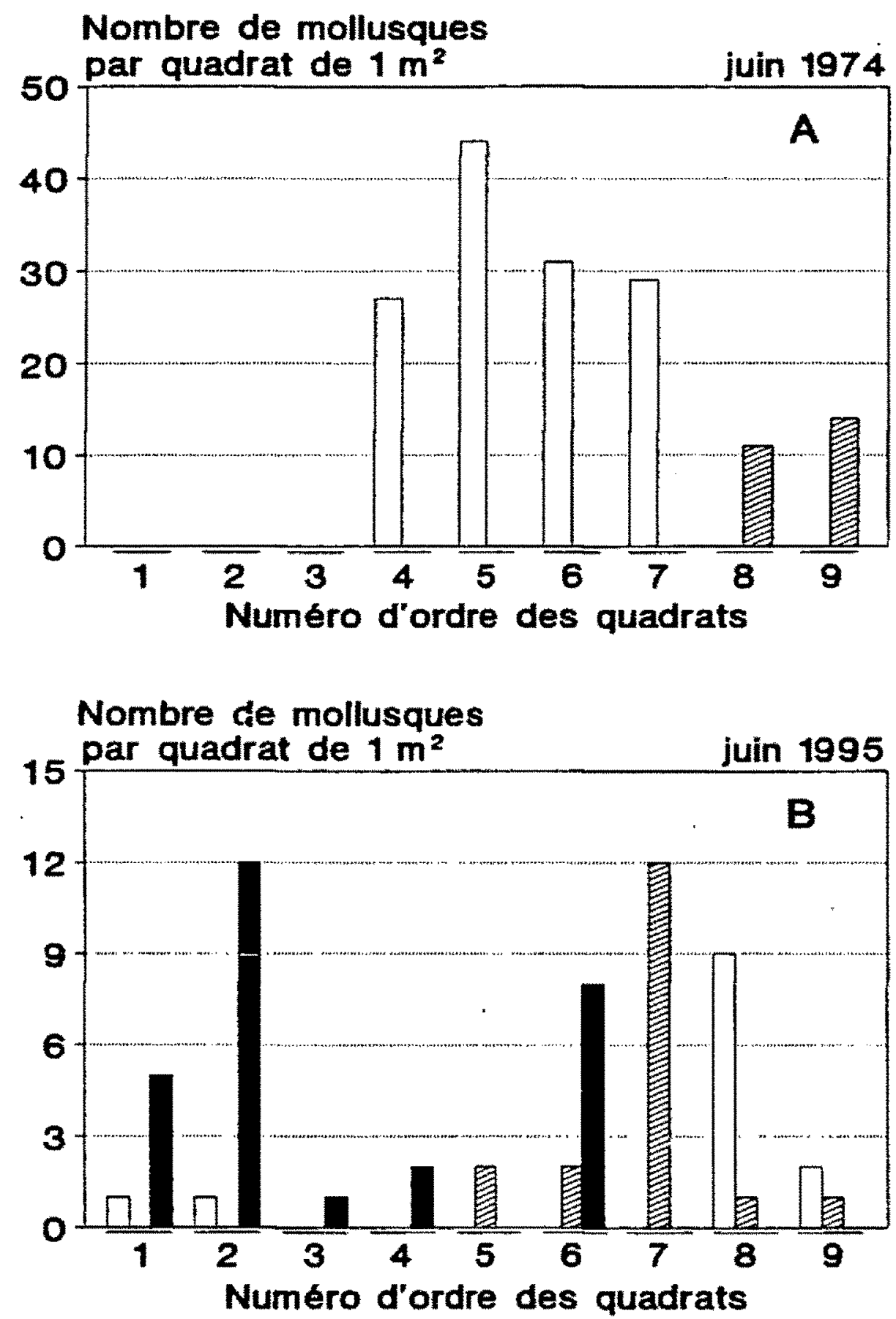

\section{Lymnaea glabra
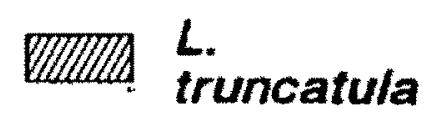 \\ $L$. ovata}

Fig. 4. Station de la Roseraie, commune de Limoges (HauteVienne). Répartition numérique des mollusques dans une rigole de drainage en juin 1974 (A) et juin 1995 (B). Les chiffres ont été obtenus sur neuf quadrats de $1 \mathrm{~m}^{2}$, choisis à intervalles réguliers sur toute la longueur de la rigole.

Fig. 4. Meadow of the Roseraie, commune of Limoges, department of Haute-Vienne, France. Numerical distribution of snails in an open drainage furrow in June 1974 (A) and June 1995 (B). Snails were counted in nine $1-\mathrm{m}^{2}$ quadrants, selected at regular intervals over furrow length.

réduction dans ses effectifs : de 320 environ en 1974 à 186 en 1995. Enfin, notons l'effectif de la physe, estimé à plus de 950 individus dans les deux fossés.

Dans la prairie de Burgnac, l'invasion de Potamopyrgus antipodarum dans la rigole de drainage s'est traduite par une extension dans la superficie des habi- tats pour les deux limnées comme pour l'espèce colonisatrice. L'aire des gîtes est de $54 \mathrm{~m}^{2}$ pour L. glabra comme pour $L$. truncatula. Par contre, on note une réduction nette dans les effectifs des limnées : $98 \mathrm{~L}$. glabra et 56 L. truncatula en 1995 au lieu de 210 et: 126 respectivement en 1974. Le prosobranche présente un accroissement numérique important : 356 individus en 1995.

Dans la prairie de Limoges, les deux espèces colonisatrices se distribuent sur $52 \mathrm{~m}^{2}$ dans le cas de Physa acuta et sur $44 \mathrm{~m}^{2}$ dans le cas de $L$. ovata. Les gîtes de $L$. glabra et de $L$. truncatula sont plus étendus en 1995 ( 30 et $37 \mathrm{~m}^{2}$ respectivement au lieu de 16 et de $12 \mathrm{~m}^{2}$ en 1974). Les deux premières espèces ont des effectifs assez importants en 1995:126 L. ovata et 316 Physa acuta. Quant à $L$. glabra et $L$. truncatula, leurs nombres ont chuté (respectivement 44 et 37 en 1995 pour 380 et 60 en 1974).

\section{Discussion}

Les perturbations sur les trois prairies ont eu plusieurs conséquences sur les colonies de $L$. truncatula et de $L$. glabra. La superficie des gîtes s'est accrue en 1995 dans les trois prairies mais la distribution de chaque espèce dans les rigoles et les couloirs reste la même, avec la plupart des Limnées tronquées à l'extrémité aveugle et de nombreuses $L$. glabra sur le cours moyen des rigoles. Les effectifs de $L$. truncatula ont nettement augmenté en 1995 dans la prairie du Châtenet. Par contre, les effectifs de cette limnée et ceux de $L$. glabra dans les autres parcelles sont à la baisse en 1995.

Le schéma proposé par Moens (1991) fait intervenir l'excès d'humidité et les perturbations du sol comme des facteurs qui favorisent la densité des populations de $L$. truncatula. Il ne s'applique que de manière imparfaite dans le cas des trois prairies et il est donc nécessaire de rechercher une autre interprétation pour expliquer ces changements. L'hypothèse la plus valable pour expliquer l'accroissement de la superficie des gîtes serait de rapporter ce fait aux difficultés qu'ont les $L$. glabra et $L$. truncatula pour trouver leur nourriture dans ces milieux envahis par d'autres mollusques, ce qui entrainerait par suite les déplacements des limnées sur une plus grande surface. Cette supposition s'appuie sur la baisse des effectifs constatée dans la plupart des cas mais elle cadre mal avec l'augmentation des effectifs pour L. truncatula dans la prairie du Châtenet. Il est difficile de rattacher cet accroissement aux fèces des ragondins car les Limnées tronquées ont été rarement observées sur ce matériau. Il est plus logique de relier ce dernier fait aux perturbations qui ré- 
Tableau 1. Les caractéristiques des gîtes à limnées dans les trois prairies modifiées par l'activité humaine. Les décimales n'ont pas été portées. * Les effectifs de Feytiat ont été établis à partir des valeurs moyennes obtenues lors des prospections de 1994.

Table 1. The characteristics of lymnaeid habitats in the three meadows modified by agricultural practices. Decimal numbers are not given. * Numbers of snails in the Feytiat meadow were calculated from mean values recorded during investigations of 1994.

\begin{tabular}{|c|c|c|c|}
\hline \multirow[b]{2}{*}{ Stations } & \multirow[b]{2}{*}{ Espèces } & \multicolumn{2}{|c|}{ Juin 1995} \\
\hline & & $\begin{array}{l}\text { Superficie } \\
\text { du gîte } m^{2} \text { ) }\end{array}$ & $\begin{array}{l}\text { Effecifs } \\
\text { estimés }\end{array}$ \\
\hline $\begin{array}{l}\text { Le Châtenet } \\
\text { (Commune de } \\
\text { Feytiat) }\end{array}$ & $\begin{array}{l}\text { L. glabra } \\
\text { L. cf. fuscus } \\
\text { L. truncatula } \\
\text { Physa acuta }\end{array}$ & $\begin{array}{r}113 \\
179 \\
89 \\
62\end{array}$ & $\begin{array}{l}186^{*} \\
873 \\
476 \\
960\end{array}$ \\
\hline $\begin{array}{l}\text { Le Marchadeau } \\
\text { (Commune de } \\
\text { Burgnac) }\end{array}$ & $\begin{array}{l}\text { L. glabra } \\
\text { L. truncatula } \\
\text { Potamopyrgus antipodarum }\end{array}$ & $\begin{array}{l}54 \\
54 \\
30\end{array}$ & $\begin{array}{r}98 \\
66 \\
356\end{array}$ \\
\hline $\begin{array}{l}\text { La Roseraie } \\
\text { (Commune de } \\
\text { Limoges) }\end{array}$ & $\begin{array}{l}\text { L. glabra } \\
\text { L. ovata } \\
\text { L. truncatula } \\
\text { Physa acuta }\end{array}$ & $\begin{array}{l}30 \\
44 \\
37 \\
52\end{array}$ & $\begin{array}{r}44 \\
126 \\
37 \\
316\end{array}$ \\
\hline
\end{tabular}

sultent des passages répétés de ces rongeurs en quête de nourriture, ce qui maintiendrait l'humidité des zones et favoriserait la croissance des algues dont se nourrissent les limnées.

Le processus le plus spectaculaire concerne $L$. cf. fuscus. En effet, cette espèce a déserté le fossé central du Châtenet pour coloniser les rigoles et les couloirs créés par les ragondins, ce qui s'est traduit par une explosion dans les effectifs du mollusque et une distribution élargie sur toute la longueur des rigoles et des couloirs. Deux hypothèses, peut-être complémentaires, peuvent expliquer ces modifications. La première serait de rapporter ce fait à la présence des crottes de ragondins car la plupart des mollusques ont été trouvés sur ces fèces ou dans leur voisinage immédiat. Il est probable que ces déjections représentent une source alimentaire pour la limnée et seule une étude du contenu intestinal par la dissection des mollusques permettrait de vérifier cette première supposition. La seconde hypothèse ferait intervenir un transport accidentel des mollusques par les ragondins pour expliquer la dissémination de la limnée sur l'ensemble des rigoles et des couloirs. Cette dernière supposition n'est pas nouvelle puisque plusieurs auteurs l'ont déjà formulée pour expliquer la colonisation de nouveaux territoires par diverses espèces de Lymnaea (Mehl 1932, Taylor 1965, Khallaayoune 1989).
Trois espèces invasives ont été retrouvées dans l'une ou l'autre de ces trois prairies. Deux d'entre elles, Physa acuta et Potamopyrgus antipodarum, sont déjà connues dans la littérature pour leur capacité à coloniser de nouvelles zones :

- La physe est une espèce invasive : l'introduction de Physa acuta en Afrique du Sud en 1954 s'est traduit par la colonisation de nombreuses rivières (De Kock et al. 1989, Brackenbury \& Appleton 1993). La présence de la physe dans $3 \%$ des habitats prairiaux de la Limnée tronquée a été également constatée par Rondelaud \& Mage (1988) mais ces auteurs n'ont pas relevé de migration active par rhéotropisme. Lors de nos observations sur le terrain, nous avons observé fréquemment des physes en train de remonter les rigoles à contre-courant mais cette invasion est limitée par le dessèchement estival si bien que le mollusque ne peut se maintenir que dans des zones qui conservent une humidité importante, même au coeur de l'été.

- Potamopyrgus antipodarum est un mollusque qui a envahi le réseau hydrographique français à partir de 1960 (Lucas 1965, Real 1973). Cette espèce a été observée également dans le Limousin par Vareille-Morel (1981, 1986). A l'heure actuelle, les colonies de Potamopyrgus sont plus limitées en étendue et se rencontrent dans certains secteurs de la Haute-Vienne comme le ruisseau du Marchadeau. Mais l'espèce résiste mal 
au dessèchement estival et c'est la raison pour laquelle nous avons constaté sa présence dans la portion aval de la rigole.

Plus surprenante est l'invasion des rigoles de la Roseraie par Lymnaea ovata. Cette espèce est habituellement confinée aux ruisseaux et rivières et sa présence n'avait pas été encore signalée dans des rigoles de drainage superficiel dans le Limousin. Certes, l'existence d'une source captée se déversant en permanence dans l'une des rigoles du réseau facilite le maintien de cette espèce dans le réseau hydrographique. On peut s'interroger sur les raisons qui ont poussé cette limnée à migrer depuis l'Aurence dans le fossé de drainage et les rigoles et il est difficile, à l'heure actuelle, d'émettre une hypothèse plausible pour expliquer ce fait.

\section{Travaux cités}

Bouix-Busson D. \& Rondelaud D. 1984. - Étude de relations entre deux espèces de limnées dans leurs habitats naturels. Rev. Méd. Vét., $135: 449.457$.

Brackenbury T.D. \& Appleton C.C. 1993. - Recolonization of the Umsindusi River, Natal, South Africa, by the invasive gastropod, Physa acuta (Basommatophora, Physidae). J. Med. Appl. Malacol., 5: 39-44.

De Kock K.N., Joubert P.H. \& Pretorius S.J. 1989. — Geographical distribution and habitat preference of the invader freshwater snail Lymnaea columella (Mollusca : Gastropoda) in South Africa. On. derst. J. Vet. Res., 56: 271-275.

Khallaayoune K. 1989. - Sheep fascioliasis in Morocco : epidemiology and serodiagnostic: $\mathrm{Ph}$. D. Thesis, Univ. Minesota, U.S.A. : 196 p.
Lucas A. 1965. - Nouvelles données sur la distribution en France d'Hydrobia jenkinsi (E.A. Smith) (Hydrobiidae, Gastéropodes). J. Conchyol., 105: 40-48.

Mage C. \& Rondelaud D. 1991. - Fasciola hepatica ou la Grande Douve chez les bovins. Inventaire de sources d'infestation des bovins au pâturage. Bull. Tech. G.T.V., $\mathrm{n}^{\circ} 396,77-83$.

Mehl S. 1932. - Die Lebensbedingungen der Leberegelschnecke (Galba truncatula Müller). Untersuchungen über Schale, Verbreitung, Lebensgeschichte, natürliche Feinde und Bekømpfungsmöglichkeiten. Arb. Bayer. Landesanst. Pflanzenbau Pflanzenschutz, 2: 1-177.

Moens R. 1991. - Factors affecting Lymnaea truncatula populations and related control measures. J. Med. Appl. Malacol., 3: 7384.

Økland J. 1990. - Lakes and snails. Environment and gastropods in 1.500 Norvegian lakes, ponds and rivers. Universal Book Services/Dr. W. Backhuys, Oegstgeest, The Netherlands : 546 p.

Real G. 1973. - Répartition en France de Potamopyrgus jenkinsi (E.A. Smith, 1889). Haliotis, 3: 199-204.

Rondelaud D. \& Mage C. 1988 . - Limnée tronquée et molluscicides. Bull. Tech. G.T.V., $\mathrm{n}^{\circ} 336,69-76$.

Taylor E.L. 1965. - Fascioliasis and the liver-fluke. F.A.O. Agricultural Studies, $\mathrm{n}^{\circ} 64,236 \mathrm{p}$.

Vareille L. 1996. - Les caractéristiques des gîtes à limnées dans le département de la Haute-Vienne. Infestation expérimentale de Lymnaea cf. fuscus Pfeiffer par Fasciola hepatica $\mathrm{L}$. Thèse Doct. Pharmacie, Université de Limoges : 122 p.

Vareille-Morel C. 1981. - Contribution à l'étude de Potamopyrgus jenkinsi (E.A. Smith, 1989). I. Biotopes et progression actuelle du Prosobranche dans les bassins supérieurs de la Dronne et de son affluent l'Isle. Ann. Stat. Biol. Besse-en-Chandesse, $15 ; 166-220$.

Vareille-Morel C. 1986. - Contribution à l'étude écologique et écophysiologique du Mollusque Prosobranche Potamopyrgus jenkinsi (E.A. Smith, 1889) dans la région Dordogne-Haute-Vienne. Thèse Doct. ès-Sci. Nat., Université de Limoges : 248 p. 